\title{
Um Modelo para Avaliar Cursos Superiores Brasileiros via Internet
}

\section{A Model to Evaluate Brazilian Higher Distance Courses by Internet}

\begin{abstract}
Resumo: Este texto apresenta um modelo para a avaliação de cursos superiores a distância via Internet, no contexto brasileiro. O processo completo de elaboração e validação do modelo proposto é apresentado de modo detalhado e sistemático. Também é apresentada a descrição da validação estatística do instrumento de avaliação proposto, por meio do cálculo do coeficiente Alfa de Cronbach. Por fim, é apresentado o sistema computacional construído, denominado SAESD - Sistema de Apoio à Avaliação de cursos do Ensino Superior a Distância -, para a coleta e a organização dos dados necessários na avaliação de um curso superior a distância com base no modelo proposto. Um resultado importante deste trabalho foi a definição de uma um conjunto de aspectos relevantes na avaliação de um curso superior a distância via Internet, a partir das quais e possível o desenvolvimento de inúmeras ferramentas computacionais para a coleta e mineração de dados sobre esses aspectos.

Palavras-chave: Coeficiente Alfa de Cronbach. Indicadores de Qualidade. Avaliação. Educação a Distância. SAESD.

Abstract: This text presents a model to evaluate higher distance courses by Internet in Brazil. The whole process of elaboration and validation of the proposal model is presented in a systematic and detailed way. It is also presented the use of Coefficient Alpha de Cronbach to statistical validation of the evaluation instrument proposed. Finally, it is presented the computational system built, named SAESD - System to support the Evaluation of Higher Distance Courses. SAESD was wroposed in this work. A relevant result of this work was the proposed in this wirk. A relevant result of this work was the definition of an important set of aspects, related to the evaluation of a higher distance course by Internet, from data collecting and data mining about these aspects. Kefficient
\end{abstract}

LACHI, Ricardo Luís; ROCHA, Heloísa Vieira da. Um Modelo para a Avaliação de Cursos Superiores Brasileiros via Internet. Informática na Educação: teoria \& prática, Porto Alegre, v. 14, n. 1, p. 79-92, jan./jun. 2011.

\author{
Ricardo Luís Lachi \\ Universidade Estadual de Mato Grosso do Sul \\ Heloísa Vieira da Rocha \\ Universidade Estadual de Campinas
}

\section{Introdução}

$\Delta$ qualidade de um curso superior, qualquer que seja a sua modalidade 1 de oferecimento (presencial, semipresencial ou a distância), é de suma importância, tanto para os alunos, quanto para quem oferece o curso. Para os alunos é importante porque há uma maior potencialidade de aprendizado e, do ponto de vista de quem oferece o curso, este é um aspecto de fundamental importância para que se tenha procura pelo curso.

No contexto brasileiro, de acordo com Sanchez (2008), um levantamento realizado pela Associação Brasileira de Educação a Distância (ABED) constatou que em 2004 foram efetivadas 159.366 matrículas em cursos de graduação e pós-graduação a distância. Também conforme Sanchez (2008), de 2003 a 2006, o número de cursos de graduação passou de 52 para 349, um aumento de $571 \%$, de acordo com levantamento realizado pelo Censo da Educação Superior do Ministério da Educação (Educacenso/INEP).

Observando a expansão da Educação a Distância (EaD), o Ministério da Educação (MEC) no ano de 2007, por meio de sua Secretaria de Educação a Distância, criou o 
documento "Referenciais de Qualidade para Educação Superior a Distância" (BRASIL, 2007), visando estabelecer princípios, diretrizes e critérios para orientar países onde é ofertada a modalidade de EaD e existem os respectivos documentos com o mesmo propósito.

No artigo de Lachi et al. (2006), um amplo estudo foi realizado sobre os processos de avaliação da qualidade na EaD no Ensino Superior em vários países como Estados Unidos, Brasil, Nova Zelândia, Reino Unido, Canadá, dentre outros. O resultado desse estudo, que incluiu o contexto brasileiro e os seus referenciais de qualidade propostos pelo MEC, foi um instrumento de avaliação que consiste em um conjunto de perguntas que auxiliam no processo de avaliação da qualidade de um curso superior a distância. As respostas coletadas para essas perguntas são parâmetros confiáveis para a avaliação da qualidade de um curso uma vez que essa confiabilidade foi atestada por meio do cálculo do coeficiente Alfa de Cronbach, que é um teste estatístico específico para avaliar a confiabilidade das perguntas de um questionário.

Ainda que um avaliador disponha desse instrumento de avaliação, a sua tarefa ainda é árdua, pois ele deve: 1) aplicar o instrumento de avaliação a cada entidade envolvida no processo educacional (professores, alunos, administradores) e coletar as respectivas respostas; e 2) efetuar a organização e análise dos dados coletados, tarefa esta sujeita a erros caso seja feita manualmente, considerando-se uma grande quantidade de respondentes. Nesse sentido a telemática pode auxiliar sobremaneira nessas tarefas por meio de um sistema de informação que auxilie o avaliador a ter dados confiáveis para julgar a qualidade de diferentes aspectos de um curso.

Assim, este texto tem a seguinte organização: na seção 2 é apresentado todo o processo de concepção do conjunto de perguntas elaboradas e o teste de confiabilidade das mesmas; na seção 3 é apresentado o SAESD (Sistema de Apoio à avaliação de cursos do Ensino Superior a Distância), um sistema computacional para auxiliar e automatizar essa tarefa; na seção instituições a criar e oferecer cursos com qualidade nessa modalidade. A elaboração desse referencial segue o exemplo de outros

4 são apresentadas as conclusões e trabalhos futuros; na seção 5 são apresentadas todas as referências bibliográficas citadas neste texto.

\section{Concepção do instrumento de avaliação}

Avaliar a qualidade de um curso a distância não é uma tarefa simples. Existem inúmeros trabalhos na literatura - Barker (1999), Bates e Poole (2003), Brasil (2007), Callejo et al. (2001), Eaton (2002), Hope (2001), Moura (2003), Phipps e Merisotis (2000), Rapchan et al. (2002), Sangrà (2001), Tait (1997) - que definem itens que são utilizados no processo de avaliação os quais podem, posteriormente, fornecer parâmetros para indicar melhorias a serem efetuadas sobre os diversos aspectos de um curso avaliado. Embora sejam utilizadas denominações diferentes para esses itens (indicadores, componentes e padrões), todos eles têm o mesmo objetivo: fornecer pontos de referência para a avaliação da qualidade de um curso a distância.

A partir da constatação desse fato, um amplo estudo foi executado e tomou-se como referência principal o trabalho de Phipps e Merisotis (2000) para a elaboração do instrumento de avaliação. Nesse documento existe um conjunto de 24 indicadores, organizados nas sete categorias seguintes:

\section{1) Suporte institucional}

- Indicador 1: existe um plano tecnológico documentado e operacional, que inclua medidas eletrônicas de segurança (i.e, proteção por senhas, criptografia, sistemas de backup), para garantir padrões de qualidade tanto para a integridade quanto para a validade das informações;

- Indicador 2: a confiabilidade do sistema tecnológico de entrega é tão infalível quanto possível;

- Indicador 3: um sistema centralizado provê o suporte para a 
construção e a manutenção da infraestrutura de educação a distância.

2) Desenvolvimento do curso

- Indicador 4: orientações relativas a padrões mínimos são utilizadas para o desenvolvimento, o desenho e a entrega de uma disciplina. Além disso, os resultados de aprendizagem são quem determinam a tecnologia a ser utilizada para a entrega do conteúdo de uma disciplina e não a disponibilidade de certa tecnologia;

- Indicador 5: os materiais instrucionais são revisados periodicamente para garantir que eles estão de acordo com os padrões do curso;

- Indicador 6: as disciplinas são projetadas para que os alunos se engajem em atividades de análise, síntese e avaliação como parte dos requisitos da disciplina e do curso.

3) Processo ensino-aprendizagem

- Indicador 7: a interação dos alunos com o corpo docente e com outros alunos é uma característica essencial e é facilitada por uma variedade de modos, incluindo $e$ mail de voz e/ou e-mail;

- Indicador 8: o feedback às atividades e às perguntas dos alunos é construtivo e dado em um tempo adequado;

- Indicador 9: os alunos são instruídos nos métodos próprios da pesquisa efetiva, dentre os quais está incluída a avaliação da validade das fontes de informação.

4) Estrutura de uma disciplina

- Indicador 10: antes de começar um curso online, os alunos são aconselhados sobre o mesmo para que possam determinar: (1) se eles têm a motivação e o comprometimento necessários para aprender a distância; (2) se eles têm acesso à tecnologia mínima definida no desenho das disciplinas;

- Indicador 11: os estudantes recebem informações suplementares sobre a disciplina explicitando a sua idéia geral, seus objetivos e os conceitos que aborda. Além disso, os resultados esperados de aprendizagem de cada disciplina estão resumidos por escrito em uma linguagem clara e direta;

- Indicador 12: os alunos têm acesso a recursos suficientes de biblioteca que pode incluir uma "biblioteca virtual" acessível pela Internet;

- Indicador 13: os professores e os alunos concordam com relação ao tempo estipulado para a resolução das atividades a serem realizadas pelos estudantes e, também concordam com relação ao tempo de resposta dos professores.

\section{5) Serviço de suporte ao aluno}

-Indicador 14: os alunos recebem informações sobre o curso, incluindo os requisitos para admissão, custos, taxas, livros, suprimentos, requisitos técnicos e disciplinares, além dos serviços de suporte existentes para o aluno;

- Indicador 15: os alunos recebem treinamento e informações que os ajudem a obter material por meio de bancos de dados eletrônicos, empréstimos em bibliotecas, arquivos governamentais, serviços de notícias e outras fontes;

- Indicador 16: por toda a duração da disciplina/curso, os alunos têm acesso a uma assistência técnica, incluindo instruções detalhadas a respeito dos meios eletrônicos utilizados, sessões práticas realizadas antes do início da disciplina e acesso fácil a equipe de suporte técnico;

- Indicador 17: as questões dirigidas ao serviço pessoal de auxílio ao aluno são respondidas rapidamente e precisamente, existindo também um sistema estruturado para receber as reclamações dos alunos.

6) Serviço de suporte ao professor

- Indicador 18: a assistência técnica para o desenvolvimento da disciplina está disponível para o corpo docente 
e eles são encorajados a fazer uso dela;

- Indicador 19: os membros do corpo docente são assistidos na transição do ensino tradicional em sala de aula para o ensino a distância e são avaliados no processo;

- Indicador 20: o treinamento e a assistência ao professor de uma disciplina a distância, incluindo a figura de um professor-mentor, também continua durante a disciplina que eles estão oferecendo online;

- Indicador 21: os professores recebem materiais escritos para auxiliá-los a lidar com questões dos alunos quanto ao acesso às informações disponíveis eletronicamente.

7) Avaliação de resultados:

- Indicador 22: a efetividade educacional do curso e o processo de ensino/aprendizagem são estimados por meio de um processo de avaliação que aplica diversos métodos e segue padrões específicos;

- Indicador 23: as matrículas, os custos e usos bem sucedidos/inovadores da tecnologia são usados para avaliar a efetividade do curso;

- Indicador 24: os resultados de aprendizagem pretendidos são regularmente revisados para garantir a clareza, utilidade e adequação dos mesmos.

A escolha desse estudo de Phipps \& Merisotis (2000) como base deste trabalho, se deu a partir do fato de que esse estudo é considerado um referencial de excelência internacional para a avaliação da qualidade de um curso a distância por meio de indicadores, conforme consta em diversas referências literatura: Barker (1999), Bates (2003), Brasil (2007), Callejo et al. (2001), Eaton (2002), Hope (2001), Moura (2003), Rapchan et al. (2002), Sangrà (2001), Tait (1997). Além disso, esse estudo também tem sido adotado como fonte principal para as pesquisas realizadas por outros trabalhos nacionais, tais como, Rapchan et al. (2002) e Moura (2003). Esse destaque que os indicadores definidos no trabalho de Phipps e Merisotis (2000) desfrutam, tanto internacionalmente, quanto nacionalmente, demonstra a grande relevância deles.

Outro ponto que reforça e endossa a escolha desse conjunto de indicadores é a sua aplicabilidade no contexto brasileiro. A dissertação de Moura (2003) já constatou a importância desses indicadores para diversas universidades nacionais e para a comunidade acadêmica brasileira de educação a distância. Esse é um ponto muito importante que deve ser ressaltado, pois implica dizer que já houve a validação das sete categorias e dos 24 indicadores do estudo de Phipps e Merisotis (2000) para o contexto brasileiro. Isto é uma premissa básica e fundamental para a adoção desse conjunto de indicadores para o projeto de um instrumento de avaliação para a educação superior a distância via Internet no Brasil.

Na Figura 1 é apresentado, de forma esquemática, todo o processo realizado para a concepção do instrumento de avaliação de cursos superiores a distância proposto neste texto.

\subsection{Elaboração do Questionário}

Inicialmente, conforme consta na Figura 1, para cada um dos 24 indicadores do trabalho de Phipps e Merisotis (2000), foi elaborado um conjunto de perguntas com base em diversas referências bibliográficas existentes na literatura, tais como, Bloom (1956), Brasil (2007), Demo (1996), Filatro (2008), Sinaes (2006), dentre outras. Cada conjunto de perguntas se originou a partir da análise e reflexão feita sobre que aspecto do curso que determinado indicador procurava avaliar.

É importante enfatizar que, além dos trabalhos já citados, inúmeros outros foram analisados e estudados para a elaboração do instrumento de avaliação, contudo ressaltase em especial a utilização do trabalho de Brasil (2007) por contextualizar a avaliação de um curso a distância no contexto nacional brasileiro. 


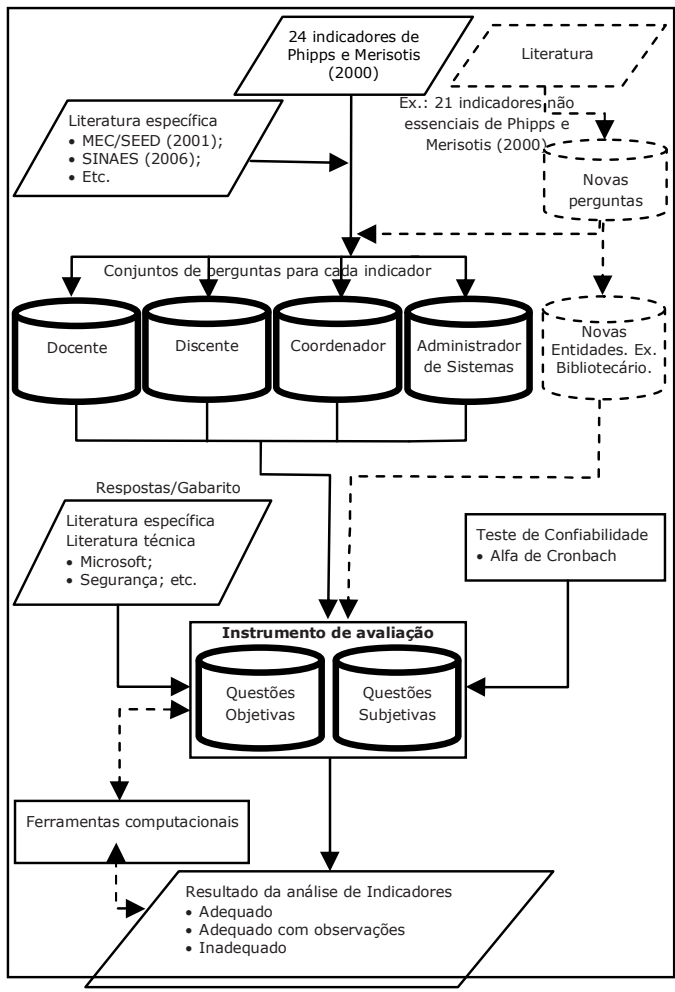

Figura 1 - Representação esquemática de todo o processo. As setas tracejadas representam as possíveis extensões que o processo realizado permite que sejam feitas.

Com base na bibliografia específica da área de cada um dos 24 indicadores listados anteriormente, cada um deles foi analisado e, com base nessa análise, foi elaborado um conjunto de perguntas específicas que permitissem a coleta de informações bem definidas a respeito de cada um deles. 0 instrumento de avaliação construído nesse processo contemplou um total de 90 questões.

A Tabela 1 apresenta um panorama geral dos quantitativos de questões elaboradas para cada um dos indicadores e categorias do trabalho de Phipps e Merisotis (2000).

Na sequência, para a coleta de respostas para cada uma das perguntas elaboradas, elas foram divididas em 4 subconjuntos, onde cada um deles deve ser respondido por uma das 4 entidades definidas para a coleta de dados junto a Instituição cujo curso está sob avaliação. As diferentes entidades envolvidas estão descritas na próxima subseção.
Tabela 1 - Listagem da quantidade de perguntas elaboradas para cada um dos 24 indicadores das 7 categorias do trabalho de Phipps e Merisotis (2000).

\begin{tabular}{|c|c|c|}
\hline Categoria & Indicador & $\begin{array}{c}\text { Quantidade de } \\
\text { perguntas }\end{array}$ \\
\hline \multirow{3}{*}{1} & 1 & 15 \\
\hline & 2 & 3 \\
\hline & 3 & 7 \\
\hline \multirow{3}{*}{2} & 4 & 2 \\
\hline & 5 & 3 \\
\hline & 6 & 4 \\
\hline \multirow{3}{*}{3} & 7 & 4 \\
\hline & 8 & 2 \\
\hline & 9 & 5 \\
\hline \multirow{4}{*}{4} & 10 & 7 \\
\hline & 11 & 6 \\
\hline & 12 & 2 \\
\hline & 13 & 2 \\
\hline \multirow{4}{*}{5} & 14 & 2 \\
\hline & 15 & 3 \\
\hline & 16 & 8 \\
\hline & 17 & 4 \\
\hline \multirow{4}{*}{6} & 18 & 2 \\
\hline & 19 & 3 \\
\hline & 20 & 1 \\
\hline & 21 & 1 \\
\hline \multirow{3}{*}{7} & 22 & 4 \\
\hline & 23 & 0* \\
\hline & 24 & $0 * *$ \\
\hline
\end{tabular}

Este indicador não tem perguntas porque foi analisado como estritamente ligado ao sistema de ensino dos EUA e portanto, desconsiderado para o contexto brasileiro.

** Relacionado à avaliação deste próprio instrumento de avaliação, portanto, fora do escopo da instituição para a coleta de informações.

\subsection{Entidades Envolvidas na Avaliação}

Neste trabalho foi identificada, para cada uma das perguntas de cada conjunto, a função da pessoa, pertencente à Instituição, que deve respondê-la. Essa organização possibilita uma clara identificação das entidades que devem ser consultadas pelo avaliador: Docente, Discente, Coordenador do Curso e Administrador de Sistemas. Na Figura 1, todas as perguntas elaboradas foram organizadas (distribuídas) entre essas quatro entidades.

Outra etapa do processo, também presente na Figura 1, é a possibilidade de inclusão de novas perguntas e/ou novas entidades, representada por meio das linhas tracejadas, que partem e chegam aos elementos "Novas perguntas" e "Novas entidades". Com isso, deseja-se deixar 
explícito que essas são possibilidades diretas de extensão do processo definido, o que o torna flexível e robusto.

\subsection{Classes de Perguntas do Questionário}

Uma vez elaboradas as perguntas e definido quem vai respondê-las, foram diferenciadas as questões objetivas das subjetivas. O primeiro tipo envolve perguntas cujas respostas não buscam coletar a opinião dos respondentes. Por exemplo, a pergunta "É possível a um participante alterar sua própria senha?" relativa ao Indicador 1 da categoria "Suporte Institucional" pode ter como resposta "sim" ou "não", que independe de opinião. Já as questões subjetivas têm respostas que envolvem a coleta de opiniões de um ou mais respondentes. Por exemplo, a pergunta "As informações que recebeu sobre os objetivos de cada disciplina estavam escritas de uma forma clara e direta?" relativa ao Indicador 2 da categoria "Estrutura de uma disciplina" coleta opiniões por meio das seguintes opções de resposta: nunca; na minoria das vezes; às vezes; na maioria das vezes; sempre. Essas opções de resposta foram definidas com base na escala de Likert $^{1}$ de 5 pontos que permite ao respondente graduar a sua concordância ou discordância com o que é perguntado.

\subsection{Teste da Confiabilidade das Questões Subjetivas}

Definidas essas duas classes de questões, buscou-se avaliar a confiabilidade de cada questão, isto é, se a resposta para uma determinada pergunta, quando coletada novamente no futuro, será replicável e consistente, ou seja, se serão obtidos resultados comparativamente equivalentes.

As questões objetivas são confiáveis, pois, tomando-se a pergunta-exemplo apresentada anteriormente, a resposta a ser

\footnotetext{
${ }^{1}$ A escala de Likert é uma escala muito utilizada para a construção de alternativas de respostas para questões de um questionário, uma vez que permite ao respondente indicar o grau de concordância ou discordância com cada uma das afirmações feitas nas questões.
}

obtida para a mesma será sempre igual, independente do momento em que ela for coletada. Por outro lado, a confiabilidade das questões subjetivas deve ser avaliada, pois elas envolvem a opinião subjetiva de uma pessoa. A avaliação da confiabilidade deste último tipo de questão foi feita por meio do cálculo do Coeficiente Alfa de Cronbach (CARMINES; ZELLER, 1979), uma vez que ele é muito utilizado na psicometria ${ }^{2}$ desde 1951 para a avaliação da confiabilidade de questões.

\subsubsection{Coeficiente Alfa de Cronbach}

Carmines e Zeller (1979) explicam que o cálculo do coeficiente Alfa de Cronbach pode ser feito por meio de uma única aplicação do instrumento de medida - no caso deste trabalho, aplicar o instrumento de medida significa coletar respostas para as questões subjetivas do questionário - produzindo valores entre 0 e 1 , ou entre 0 e $100 \%$.

A expressão do coeficiente Alfa de Cronbach é dada por:

$$
\begin{aligned}
& \alpha=\frac{N \bar{p}}{\lceil 1+\bar{p}(N-1)\rceil} \\
& \text { Onde, } \\
& \qquad \begin{array}{l}
\mathrm{N}=\text { número de itens; } \\
\bar{p}=\text { média dos coeficientes de } \\
\text { correlação linear (Pearson) entre os } \\
\text { itens; } \\
\quad 0 \leq \alpha \leq 1 \text { ou } 0 \leq \alpha \leq 100 \% .
\end{array}
\end{aligned}
$$

Para avaliar a confiabilidade das questões subjetivas propostas, foram coletadas respostas de 134 alunos de um curso superior a distância para cada uma dessas questões e, a partir das respostas obtidas, foi utilizado o software estatístico SPSS 17.0.0 $0^{3}$ para o cálculo do coeficiente Alfa de Cronbach.

As perguntas elaboradas para um determinado indicador foram mantidas no instrumento de avaliação caso o Alfa calculado para elas, a partir das respostas

\footnotetext{
2 Psicometria: medida da duração e da intensidade de processos mentais, por meio de métodos padronizados (definição extraída do "Novo Dicionário Eletrônico Aurélio 5.0")

${ }^{3}$ http://www-01.ibm.com/software/analytics/spss/.
} 
coletadas, tenha sido maior que 0,6. Isso porque, de acordo com Pestana e Gageiro (2003), acima desse valor as perguntas já são aceitáveis, isto é, as questões mantidas já podem ser consideradas confiáveis para medir o indicador a que estão associadas.

\subsubsection{Tamanho da Amostra Considerada}

Já com relação ao tamanho da amostra utilizada ser suficiente, Fink (1995) em seu livro How to sample in surveys, afirma que o tamanho de uma amostra se refere ao número de respondentes necessário para que os resultados obtidos sejam precisos e confiáveis e que o aumento do tamanho da amostra diminui o erro. Naturalmente, essa tendência tem limites; a partir de certa quantidade não se tem mais uma forte contribuição agregada por coletar-se maior número de respostas a um questionário.

Moscarola (1990) apresenta que com uma amostra inferior a 30 observações se tem chances de encontrar tanto um valor errôneo ou defasado como um valor próximo da realidade. E que as chances de obtenção de valores ou resultados mais próximos da realidade aumentam bastante com pelo menos 100 observações. Artes (1998), Kline (1994) e Menezes (1998) são outros autores que também reforçam a idéia de que pelo menos 100 observações devam ser feitas.

Portanto, conforme mostra a literatura, o tamanho da amostra considerado, de 134 respondentes, é suficiente para se encontrar valores que reflitam a realidade.

\subsubsection{Cálculo do Alfa de Cronbach}

Como exemplo da análise realizada para a avaliação da confiabilidade das questões elaboradas, na Tabela 2 é apresentada a listagem das questões subjetivas elaboradas para o indicador 1 da categoria "Estrutura de uma disciplina".

Com relação às questões listadas na Tabela 2, deve-se destacar que há uma relação de "dependência" essas questões: a questão 2 depende da questão 1 ; as questões 4 e 5 dependem da questão 3; a questão 7 depende da questão 6. Essa relação de dependência deve ser entendida como o fato de só haver sentido um determinado respondente responder a uma pergunta que é dependente, no caso dele ter dado uma resposta afirmativa à questão a qual essa pergunta é dependente.

Tabela 2 - Questões do "Indicador 1" da categoria "Estrutura de uma disciplina".

\section{Indicador 1}

1. Você recebeu um material (guia) onde constassem os direitos, deveres e atitudes de estudo necessárias para fazer o curso a distância?

2. De que forma foi disponibilizado esse material com as orientações?

3. Você recebeu um material onde constasse o comprometimento necessário para fazer o curso a distância?

4. De que forma foram passadas essas orientações?

5. Quais dos tópicos a seguir foram abordados nessas orientações disponibilizadas a você relativas ao seu comprometimento?

6. No material de divulgação do curso a distância aparecem especificados os requisitos de equipamento mínimos exigidos de você para a realização do mesmo?

7. Nesse material de divulgação quais dos seguintes itens técnicos aparecem especificados?

Consequentemente, o coeficiente Alfa de Cronbach será calculado somente sobre as respostas obtidas para as questões 1, 3 e 6, que são independentes. Isso porque, uma vez eliminada/mantida uma dessas questões independentes, automaticamente, a respectiva questão dependente será eliminada/mantida.

Na Tabela 3 é apresentado o cálculo do coeficiente Alfa de Cronbach efetuado pelo software estatístico SPSS.

Os dados apresentados na Tabela 3 foram personalizados para conter aqueles que são os mais relevantes para o objetivo da análise requerida neste trabalho.

Na Tabela 3, a sub-tabela intitulada "Inter-Item Correlation Matrix" apresenta uma informação fundamental: se as correlações entre os itens (questões) são positivas. Essa é uma premissa fundamental para a aplicabilidade do cálculo do coeficiente de Cronbach, uma vez que a existência de uma correlação negativa viola o modelo matemático subjacente ao 
coeficiente de Cronbach ${ }^{4}$.

Como no caso das questões desse indicador todas as correlações foram positivas, não houve a necessidade de nenhum ajuste e, portanto, o valor do coeficiente Alfa de Cronbach calculado é válido.

Tabela 3 - Relatório gerado pelo SPSS.

\begin{tabular}{||l|c|c|c|}
\hline \multicolumn{4}{|l|}{ Inter-Item Correlation Matrix } \\
\hline & questao1 & questao3 & questao6 \\
\hline questao1 & 1,000 &, 393 &, 322 \\
\hline questao3 &, 393 & 1,000 &, 547 \\
\hline questao6 &, 322 &, 547 & 1,000 \\
\hline
\end{tabular}

\section{Item-Total Statistics}

\begin{tabular}{|l|c|c|c|}
\hline & $\begin{array}{c}\text { Scale } \\
\text { Mean if } \\
\text { Item } \\
\text { Deleted }\end{array}$ & $\begin{array}{c}\text { Scale } \\
\text { Variance } \\
\text { if Item } \\
\text { Deleted }\end{array}$ & $\begin{array}{c}\text { Cronbach's } \\
\text { Alpha if } \\
\text { Item } \\
\text { Deleted }\end{array}$ \\
\hline questao1 & 6,29 & 4,401 &, 700 \\
\hline questao3 & 6,50 & 3,640 &, 483 \\
\hline questao6 & 6,08 & 4,568 &, 564 \\
\hline
\end{tabular}

\section{Reliability Statistics}

\begin{tabular}{|c|c|c|}
\hline $\begin{array}{c}\text { Cronbach's } \\
\text { Alpha }\end{array}$ & $\begin{array}{c}\text { Cronbach's } \\
\text { Alpha Based } \\
\text { on } \\
\text { Standardized } \\
\text { Items }\end{array}$ & $\begin{array}{c}\text { N of } \\
\text { Items }\end{array}$ \\
\hline, 682 &, 686 & 3 \\
\hline
\end{tabular}

$\mathrm{Na}$ sub-tabela intitulada "Item-Total Statistics" são apresentadas 3 colunas: a) "Escala Média, se o Item é desprezado": indica a questão que tem o maior peso para o indicador, no caso, a questão 3 ; b) "Variância da Escala, se o Item é desprezado": indica a questão que tem a maior variabilidade na medida, no caso, a questão 6; c) "Alfa de Cronbach, se o Item é desprezado": representa o impacto que teria a retirada de cada questão sobre o

4 Para se manter uma variável que tenha apresentado uma correlação negativa, seu sentido de direção deve ser mudado, isto é, os valores numéricos atribuídos para as respostas dessa questão devem ser multiplicados pelo valor -1 . desempenho do indicador, no caso, a questão 1 é a que causaria mais impacto.

Particularmente, o valor apresentado na coluna "c" dessa sub-tabela "Item-Total Statistics" é importante de ser confrontado com o valor do Alfa de Cronbach apresentado na $1^{\text {a }}$ coluna mais à esquerda da sub-tabela intitulada "Reliability Statistics" $^{\prime 5}$. Com esse objetivo, foi construída a Tabela 4.

Tabela 4 - Cálculo do valor de Alfa para as questões da categoria "Estrutura de uma disciplina".

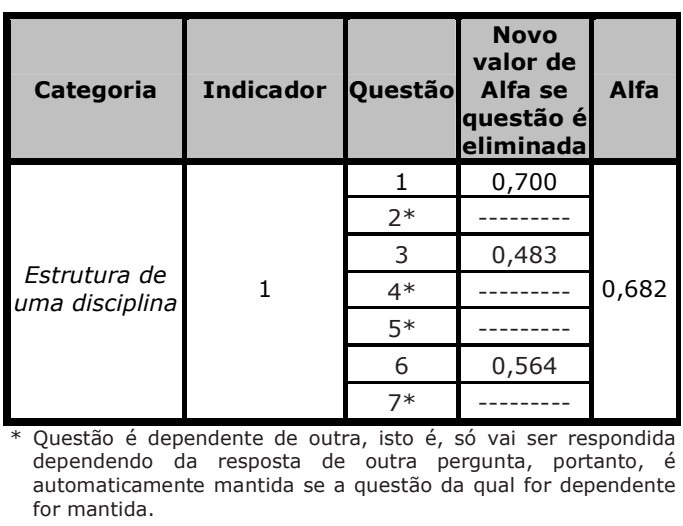

Como pode ser observada na Tabela 4, a eliminação de qualquer uma das questões, ou reduziria o valor de alfa calculado para elas, ou ocasionaria um aumento muito pequeno $^{6}$ no valor de Alfa (já está com um valor de consistência interna bem superior ao mínimo desejado: valor maior ou igual a $0,6)$, o que reforça o fato de que todas as questões devem ser mantidas.

Além disso, conforme já explicado, as questões marcadas com um asterisco são questões "casadas" com outras. Especificamente, a questão 2 é dependente da questão 1 ; a questão 4 e 5 são

\footnotetext{
5 Nota-se que também é apresentado um valor de Alfa "padronizado", que se refere à fixação da variação interna de cada medida à unidade (transformação das medidas em $z$-scores). No exemplo, Alfa e Alfapadronizado têm valores muito semelhantes porque todas as medidas se utilizaram de uma mesma escala (valores de 1 a 5 atribuídos as respostas dessas questões) e com variâncias razoavelmente próximas, (variâncias das questões 1,3 e 6 são, respectivamente, iguais a 1,590, a 1,690 a 1,171) resultando em uma variância média próxima da unidade (valor igual a 1,483).

6 No caso o aumento, com 4 casas de aproximação decimal, é de 2,6392\% (dois vírgula seis mil trezentos e noventa e dois por cento). Esse é o resultado do seguinte cálculo: $100 *(0,700 / 0,6820-1)$.
} 
dependentes da questão 3; e a questão 7 é dependente da questão 6 . Com base nesse "casamento", todas as questões desse "Indicador 1 " serão mantidas no modelo, uma vez que todas aquelas de quem dependem também serão mantidas;

Com base nesse exemplo e no conceito matemático por trás do coeficiente Alfa de Cronbach, duas considerações importantes podem ser tecidas:

1) A primeira consideração se relaciona com a perspectiva da generalização dos resultados particulares deste estudo para o universo ao qual se refere: como o valor de Alfa calculado foi de 0,682, isso significa que 0 conjunto de perguntas elaborado representa $68,2 \%$ do universo de possíveis perguntas que podem ser elaboradas para o indicador, caso alguém fosse elaborar outro conjunto de perguntas em igual quantidade;

2) A segunda consideração é consequência direta da própria natureza de "reprodutibilidade" de resultados que o coeficiente Alfa de Cronbach atesta: mesmo se outro conjunto de questões fosse elaborado para o mesmo indicador, ainda assim as medidas deste outro conjunto de questões concordariam com as daquele conjunto em $68,2 \%$ das vezes.

Essas considerações são válidas também para todas as demais questões subjetivas, uma vez que a análise feita para todas as demais questões subjetivas elaboradas foi rigorosamente a mesma do exemplo dado.

Concluindo, os questionários finais, após o cálculo do coeficiente Alfa de Cronbach e a consequente eliminação das questões subjetivas que não atingiram o nível mínimo de confiabilidade (valor maior ou igual a $0,6)$, estão disponíveis no seguinte endereço: https://Www. comp.uems.br/ Members/rlachi/2011/CITA2011/Apendice 20E.pdf/download.

\subsection{Resultado da Análise de um Indicador}

A Figura 1 mostra que as opções de respostas para ambas as classes de questões foram extraídas da literatura técnica e/ou específica relacionada diretamente ao contexto abordado em cada uma das questões, garantindo com isso, a pertinência desse conjunto de opções.

Com base nesse gabarito e nas respostas obtidas para cada uma das perguntas que compõem o questionário construído, que é feita a classificação final de um determinado indicador: "Adequado", "Adequado com observações" e "Inadequado".

\subsection{Extensões}

Por fim, a Figura 1 destaca, por meio de uma linha tracejada, a possibilidade de construção de ferramentas computacionais para facilitar o trabalho do avaliador em dois aspectos: 1) na aplicação e na coleta de respostas do instrumento de avaliação concebido; e 2) na automatização da avaliação final de cada um dos indicadores.

Visando possibilitar a coleta, a administração e a análise dos dados relevantes para a avaliação de um curso a distância via Internet foi construído o SAESD (Sistema de Apoio à Avaliação de Cursos do Ensino Superior a Distância). Esse sistema foi desenvolvido com o objetivo de facilitar esses dois aspectos apontados e também servir como uma plataforma base para a integração de novas ferramentas para a mineração dos dados registrados em um ambiente de Educação a Distância via Internet.

\section{O Sistema de Apoio à Avaliação de Cursos do Ensino Superior a Distância}

O Sistema de Apoio à Avaliação de Cursos do Ensino Superior a Distância (SAESD) abrange uma interface específica para a coleta de dados (questionários) e outra que contempla a apresentação dos dados coletados (relatórios).

\subsection{Interface de Coleta de Dados}

A coleta de dados é realizada sobre as entidades docente, discente, coordenador e administrador do sistema de e-learning ${ }^{7}$.

\footnotetext{
${ }^{7}$ Sistemas como Moodle, TelEduc, Amadeus etc.
} 
Cada entidade responde um questionário com perguntas específicas.

Para a implementação eletrônica dos questionários foram pesquisados diversos programas geradores de formulários, dentre os quais, pode-se destacar o Quiz Faber (http://lucagalli.net/en/index.php) e o Hot Potatoes (http://hotpot.uvic.ca/index.php), por serem amplamente utilizados. Contudo, após uma análise inicial desses programas, foi feita a constatação de que ambos não atendiam plenamente as necessidades do sistema a ser construído, uma vez que, nenhum desses programas prevê a possibilidade de serem definidas dependências entre duas ou mais perguntas de um questionário.

Por exemplo, suponha que um questionário tenha as perguntas $X$ e $Y$, onde a pergunta $X$ contém $O$ conjunto de alternativas ' $a$ ', $b$ ' e ' $c$ '. A partir desse cenário, deseja-se definir que $Y$ seja dependente das alternativas ' $a$ ' $e$ ' $b$ ' da questão $X$. Em outras palavras, a questão $Y$ somente deve ser exibida ao respondente para que este a responda, nos casos em que o respondente tenha selecionado como resposta da questão $X$, uma das alternativas 'a' ou 'b' desta questão.

Como esse tipo de necessidade não era coberto até o momento pelos programas citados e os questionários construídos necessitam dessa característica, haveria dificuldades na utilização direta desses programas, uma vez que, implicaria na realização de adaptações e esforços extras, caso fossem utilizados.

Além disso, no caso específico do Quiz Faber, embora seja um programa de código aberto, foi desenvolvido em $\mathrm{C}++$ e a realização de modificações no seu código, além de ser relativamente complexa, poderia prejudicar a portabilidade do questionário gerado e exigiria um esforço não desprezível para que isso não ocorresse $^{8}$, não reduzindo, portanto, o esforço empregado na criação de um questionário. Já o Hot Potatoes não tem código aberto, o que inviabiliza a realização

\footnotetext{
${ }^{8}$ Seria necessário gerar o binário executável para mais de uma plataforma - Linux, Windows XP, Windows 7, por exemplo -, testar as modificações feitas e eliminar quaisquer erros que aparecessem.
}

de modificações no mesmo. Desse modo, cada um dos questionários do SAESD foi completamente implementado com as linguagens HTML, Javascript e AJAX, usando folhas de estilo (Cascading Style Sheets).

Os questionários de todas as entidades têm basicamente os mesmos elementos (Figura 2): a) um texto identificador da entidade a que se refere o questionário; b) um texto indicativo da categoria a qual pertence as perguntas que são apresentadas na tela; c) o conjunto de questões que devem ser respondidas; d) botões com as ações possíveis de serem executadas no momento: "Continuar" que leva a outra tela" contendo as questões da próxima categoria, caso ainda tenham outras questões para serem respondidas; "Sair" que retorna a tela de acesso inicial ${ }^{10}$.

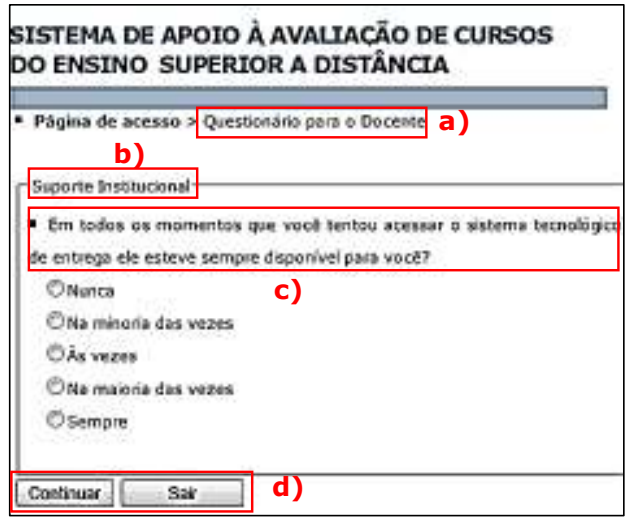

Figura 2 - Tela ilustrativa do questionário a ser preenchido.

Concluindo, por questões do espaço disponível neste artigo, não são apresentadas na Figura 2 todas as perguntas que devem ser respondidas pelo docente (total de 17 perguntas) e nem tão pouco, as interfaces dos questionários das outras entidades. Assim, como o foco desta

\footnotetext{
${ }^{9}$ O sistema está programado para permitir a ida para a próxima tela somente após terem sido respondidas todas as questões da tela atual. Caso esse preenchimento não tenha sido feito, ao clicar sobre esse botão, o sistema continuará na tela atual e destacará todas as questões ainda não preenchidas.

${ }^{10}$ As respostas preenchidas até o momento anterior ao clique no botão "Sair", já estarão salvas; isso porque o sistema foi programado de modo que, ao ser selecionada uma resposta para uma determinada questão, a mesma é automaticamente salva no banco de dados Mysql por meio da execução de uma consulta AJAX.
} 
seção é fundamentalmente a apresentação da interface construída, a Figura 2 apresentada serve como ilustração representativa, uma vez que as interfaces dos demais questionários seguem o mesmo padrão.

\subsection{Interface de Apresentação dos Dados Coletados}

O avaliador tem ao seu alcance, de modo centralizado, as respostas coletadas para as perguntas de todos os questionários, organizadas de acordo com as categorias e o conjunto de indicadores de qualidade.

Na Figura 3 é apresentada a tela inicial onde o avaliador tem a sua disposição a listagem das 7 categorias. A divisão em categorias é uma questão meramente organizacional, já que elas não são avaliadas; apenas os indicadores são. Isso decorre do fato que Phipps e Merisotis (2000) fazem questão de explicitar em seu estudo: que a definição das categorias foi somente uma forma de organização dos indicadores propostos, com base nas principais funções existentes em qualquer Instituição, sem qualquer objetivo além deste.

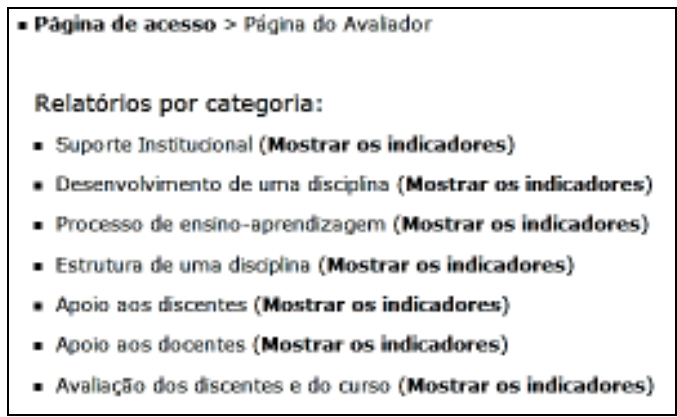

Figura 3 - Relatório disponível para o avaliador.

Nessa tela da Figura 3, o avaliador tem a sua disposição links rotulados "Mostrar os indicadores"11. Ao clicar sobre um deles, o conjunto de indicadores que compõem a respectiva categoria fica visível (Figura 4).

\footnotetext{
$11 \mathrm{Na}$ área de Interface Humano Computador (IHC) essa opção representa o conceito intitulado "informação sob demanda". A existência dessa opção é consistente com o que Shneiderman (1998) define para a visualização de informações: fornecer uma visão geral primeiro e então detalhar sob demanda.
}

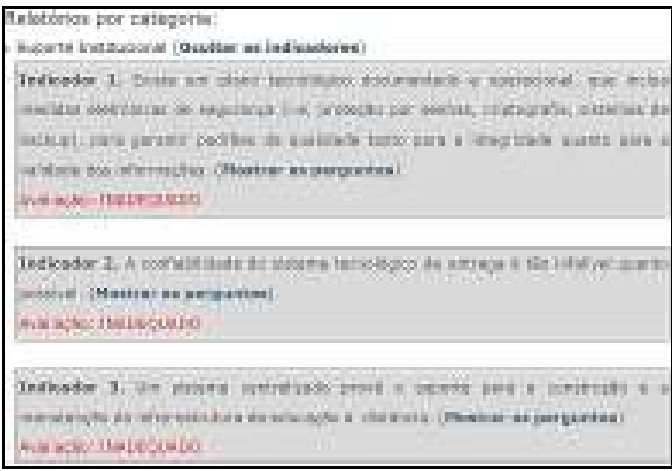

Figura 4 - Tela com as informações de indicadores da categoria "Suporte Institucional".

Na Figura 4, podem ser vistos os três indicadores que compõem a categoria Suporte Institucional e, para cada um deles, é apresentado um parecer baseado nas respostas obtidas para as perguntas correspondentes. Nessa figura, os 3 indicadores foram qualificados como 'Inadequados'.

As qualificações dos indicadores são atribuídas como descrito a seguir:

- Adequado: quando as respostas coletadas para todas as perguntas que compõem o indicador, recebem a qualificação "Adequada";

- Adequado com observações: quando as respostas coletadas para todas as perguntas que compõem o indicador, ou recebem a qualificação "Adequada", ou recebem a qualificação "Adequada com observações";

- Inadequado: caso uma das perguntas do indicador receba uma qualificação "Inadequada".

Ao lado de cada indicador, o avaliador dispõe do link 'Mostrar as perguntas' para ver mais detalhadamente o porquê do parecer atribuído a um determinado indicador. Ao clicar sobre esse link são tornados visíveis os dados coletados para todas as perguntas do respectivo indicador (Figura 5).

Na Figura 5 pode-se ver que são listadas as três perguntas específicas do Indicador $\mathbf{2}$ da categoria Suporte Institucional. Ao lado de cada pergunta sempre é apresentada uma imagem de um ' $X$ ', 'Exclamação' ou 'Certo' para indicar se os 
dados coletados para a pergunta resultam respectivamente em:

- Adequado: se acima de $60 \%$ dos respondentes tiver respondido positivamente ${ }^{12}$ a respectiva questão;

- Adequado com observações: se entre $40 \%$ e $60 \%$ dos respondentes tiver respondido positivamente a respectiva questão;

- Inadequado: no caso em que menos de $40 \%$ dos respondentes tiverem respondido positivamente a questão.

Os limites percentuais de cada um desses intervalos (acima de 60\%, entre 40\% e $60 \%$; menos de 40\%) foram definidos com base nos limites que o MEC estipula para os itens que avalia no SINAES: 0-2: muito ruim; 2-4: ruim; 4-6: regular; 6-8: bom; 810: muito bom. Novamente, é importante ressaltar essa contextualização para o caso específico brasileiro.

Como pode ser visto, é possível identificar três intervalos "conceituais" a partir desses 5 intervalos definidos pelo MEC: um intervalo, compreendendo as notas de 0 a 4, que representa itens com avaliação "conceitualmente" inadequada; um intervalo, compreendendo as notas de 4 a 6, que representa itens com avaliação "conceitualmente" adequada, mas que podem ser melhorados; um intervalo, compreendendo as notas de 6 a 10, que representa itens com avaliação "conceitualmente" adequada.

A partir desses três intervalos "conceituais" foram definidas as três qualificações para as respostas coletadas para as perguntas do instrumento.

Como pode ser visto na Figura 5, as perguntas 1,2 e 3 receberam respectivamente as marcações " $X$ ", " $X$ " e "Certo". Nessa figura, além dessas marcações, são apresentadas para cada uma das perguntas as seguintes informações: o seu enunciado; a resposta obtida; e quem a respondeu. Em relação à resposta coletada para a pergunta também é disponibilizado um link, intitulado "ver detalhes", que abre uma janela contendo

12 Uma resposta é considerada como tendo sido respondida positivamente quando foi marcada, ou a opção "Sempre", ou "Na maioria das vezes". todos os quantitativos de respostas obtidas para a pergunta.

Por fim, nas situações em que uma pergunta recebe uma marcação " $X$ " ou "Exclamação", também é apresentada uma mensagem enfatizando a necessidade de adequação da respectiva questão (mensagens destacadas em amarelo e em vermelho na Figura 5).

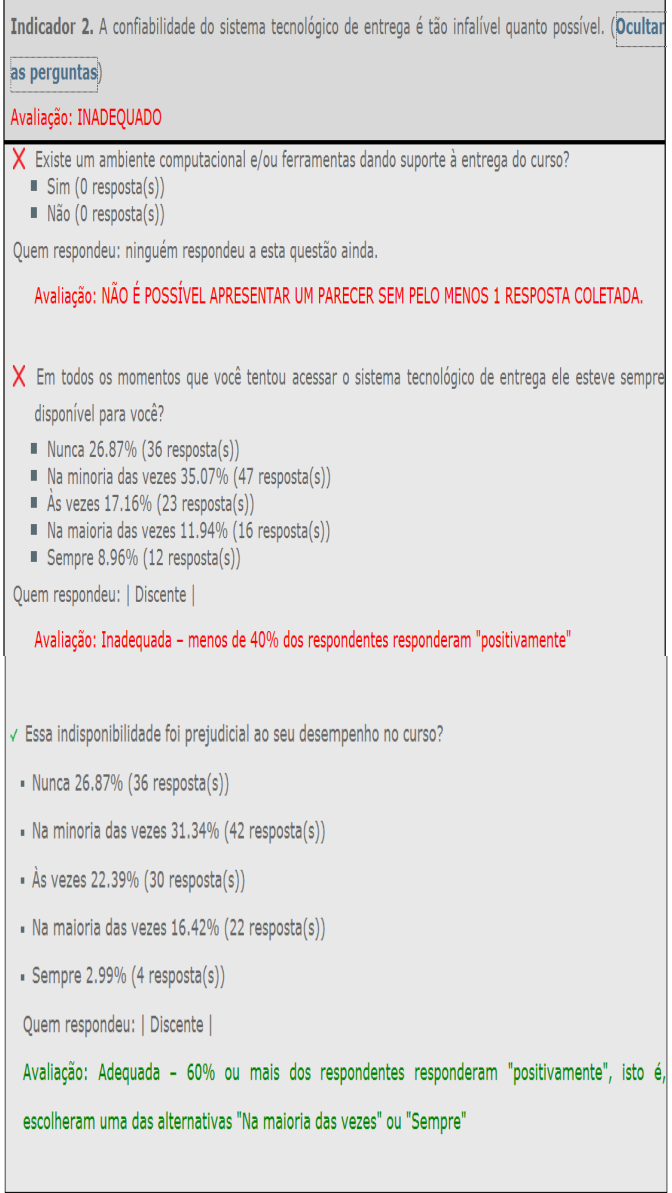

Figura 5 - Tela com a visualização dos dados coletados para cada uma das perguntas do indicador 2 da categoria "Suporte Institucional".

\section{Conclusões e Trabalhos Futuros}

Uma das grandes dificuldades existentes na avaliação de um curso à distância envolve justamente a identificação, mapeamento e compilação das bibliografias específicas mais relevantes que devem ser observadas e analisadas para o início dos trabalhos. Nesse sentido, este trabalho cita 
um subconjunto bastante relevante que pode e deve ser consultado inicialmente por pesquisadores interessados na área; com um destaque especial para os estudos de Brasil (2007) e Phipps e Merisotis (2000).

Este trabalho também apresenta a descrição explícita de um processo bem definido para se conseguir efetivamente obter um instrumento para a avaliação da qualidade de um curso a distância, com a validação da confiabilidade do instrumento obtido por meio de um teste estatístico específico e adequado a essa finalidade.

Sobre o teste estatístico realizado, dois pontos relevantes devem ser ressaltados: 1 ) o cálculo do coeficiente Alfa de Cronbach traz o rigor e o formalismo matemático na comprovação da confiabilidade do instrumento elaborado; 2) a utilização dessa prova estatística implica diretamente no aumento da relevância do instrumento obtido que pode ser usado como parâmetro comparativo com outros que sigam o mesmo processo de construção.

Por fim, é importante deixar claro que o objetivo principal do instrumento elaborado e do sistema computacional construído para dar suporte a esse instrumento, não é o de apresentar em um veredito definitivo, "bom" ou "ruim", sobre um curso. Como pode ser visto no sistema construído e durante todo o texto, o objetivo é sempre o de apresentar os pontos positivos concomitantemente com os aspectos que ainda podem ser aprimorados no curso sob avaliação.

Com relação aos trabalhos futuros, duas grandes linhas de pesquisa se descortinam a partir deste trabalho:

- Análise de todas as perguntas elaboradas, visando a pesquisa de quais informações seriam importantes que um ambiente de Educação a Distância (EaD) registrasse, de modo que possam ser recuperadas facilmente a posteriori. Essa linha de pesquisa deve contribuir para orientações sobre a especificação de arquiteturas de ambientes de EaD, a partir da indicação de quais informações esses ambientes deveriam registrar;

- Análises de todas as perguntas elaboradas, visando a pesquisa de quais outras ferramentas podem ser construídas para a coleta de dados automaticamente a partir de um ambiente de EaD.

\section{Referências}

ARTES, R. Aspectos estatísticos da análise fatorial de escalas de avaliação. Revista de Psiquiatria Clínica, 25(5), p. 223-228, 1998.

BARKER, K. Quality guidelines for technology assisted distance education. FuturEd. 1999. Disponível em: <http://www.futured.com/pdf/distance.pdf>. Acesso em: 15 fev. 2011.

BATES, T.; POOLE, G. Effective Teaching with Technology in Higher Education: Foundations for Success. Jossey-Bass. 2003. 336p. Disponível em: <http://www.batesand poole.ubc.ca/theBook.html>. Acesso em: 14 fev. 2011.

BLOOM, B. S. Taxonomy of Educational Objectives, the classification of educational goals - Handbook I: Cognitive Domain. New York: McKay. 1956.

BRASIL. Secretaria de Educação A Distância - MEC. REFERENCIAIS DE QUALIDADE PARA EDUCAÇÃO SUPERIOR A DISTÂNCIA. 2007. Disponível em: <http://portal. mec.gov.br/seed/arquivos/pdf/legislacao/ refead1.pdf>. Acesso em: 20 mar. 2011.

CALLEJO, J.; AGUADO, T.; BALLESTEROS, B.; JAURENA, I. G.; LÓPEZ, B. Indicadores de la evaluación de la educación a distancia en un sistema universitario. Revista iberoamericana de Educación a Distancia, Madrid, v.4,n.1,p.35-50, jun. 2001. Disponível em: <http://www.utpl.edu.ec/ried/index.php? option=com_content\&task=view\&id=39\&Itemid=53>. Acesso em: 18 mar. 2011.

CARMINES, E. G.; ZELLER, R. A. Reliability and Validity Assessment. Beverly Hills, CA: Sage, 1979. 
DEMO, P. Educação e Qualidade. 10.ed. Campinas: Papirus, v. 1, 1996. 160 p.

EATON, J. S. Maintaining the delicate balance: distance learning, higher education accreditation, and the politics of self-regulation. ACE - American Council on Education, Washington, DC. 2002.

FILATRO, A. Design Instrucional na prática. São Paulo: Pearson Education do Brasil. 2008. 187 p.

FINK, A. How to sample in surveys. Second Edition. 1995. ISBN: 9780761925774.

HOPE, A. Quality Assurance. In: FARRELL, G. (ed.). The Changing Face of Virtual Education. Vancouver: Commonwealth of Learning. 2001. Disponível em: <http://www.Igfl.net/imfundo/web/tech/documents /changingface/chapter7.pdf>. Acesso em: 15 fev. 2011.

KLINE, P. An easy guide to factor analysis. London: Routledge. 1994.

LACHI, R. L.; OEIRAS, J. Y. Y.; ROCHA, H. V. da. Avaliação de cursos a distância: uso de indicadores para assegurar qualidade. XVII Simpósio Brasileiro de Informática na Educação - SBIE2006. Brasília, 2006.

MENEZES, P. R. Validade e confiabilidade das escalas de avaliação em psiquiatria. Revista de Psiquiatria Clínica, 25(5), p. 214-216, 1998.

MOSCAROLA, J. Enquêtes et analyse de données. Paris, Vuibert, 1990. 307p.

MOURA, S. L. Indicadores de qualidade de cursos a distância baseados na internet. Dissertação (Mestrado) - Faculdade de Educação, Universidade Católica de Petrópolis, Rio de Janeiro. 2003.

PESTANA, M. H.; GAGEIRO, J. N. Análise de dados para as ciências sociais. Lisboa: Edições Sílabo, 2003.

PHIPPS, R.; MERISOTIS, J. Quality on the line - benchmarks for success in Internet-based distance education. Washington, DC: The Institute for Higher Education Policy. 42p (Relatório). 2000.

RAPCHAN, F. J. C.; CURY, D.; MENEZES, C.; FALBO, R. de A. EduQNet: Um Modelo de Qualidade de Processo para Cursos a Distância Mediados pela Internet. Simpósio Brasileiro de Qualidade de Software. Gramado, 2002.

SANCHEZ, F. Instituto Monitor (Org.). Anuário Brasileiro Estatístico de Educação Aberta e a Distância: ABRAED- 2008. São Paulo: Monitor, 2008.

SANGRÀ, A. La calidad em lãs experiências virtuales de educación superior. Cuadernos IRC, 2001. Disponível em: http://www.uoc.edu/web/esp/art/uoc/0106024/sangra.html. Acesso em: 20 mar. 2011.

SINAES. Avaliação externa de instituições de educação superior - Diretrizes e Instrumento. MINISTÉRIO DA EDUCAÇÃo. Comissão Nacional de Avaliação da Educação Superior. Instituto Nacional de Estudos e Pesquisas Educacionais Anísio Teixeira. 2006.

TAIT, A. International perspectives on quality assurance in open and distance learning: the importance of context. In: TAIT, Allan (ed.). Quality assurance in higher education: selected case studies. Vancouver: Commonwealth of Learning, 1997. 98p.

Recebido em maio de 2011

Aprovado para publicação em junho de 2011

\section{Ricardo Luís Lachi}

Professor da área de Ciência da Computação da Universidade Estadual de Mato Grosso do Sul - UEMS, Dourados - Brasil. E-mail: ricardo@comp.uems.br.

Heloísa Vieira da Rocha

Professora do Instituto de Computação da Universidade Estadual de Campinas - UNICAMP, Campinas Brasil. E-mail:heloisa@ic.unicamp.br. 